\title{
Acute Respiratory Distress Syndrome due to Strongyloides stercoralis Infection in a Patient with Cervical Cancer
}

\author{
Takeshi Kinjo ${ }^{1}$, Daijiro Nabeya ${ }^{1}$, Hideta Nakamura ${ }^{1}$, Shusaku Haranaga ${ }^{1}$, Tetsuo Hirata ${ }^{1}$, \\ Tomoko Nakamoto $^{2}$, Eriko Atsumi ${ }^{1,3}$, Tatsuya Fuchigami ${ }^{4}$, Yoichi Aoki ${ }^{2}$ and Jiro Fujita ${ }^{1}$
}

\begin{abstract}
A 62-year-old woman complained of diarrhea and vomiting after receiving chemotherapy for cervical cancer in association with high doses of corticosteroids. Two months later, the patient developed acute respiratory distress syndrome, and numerous Strongyloides stercoralis parasites were found in the intrabronchial discharge. Ivermectin was administered daily until nematodes were no longer detected in the sputum, and the patient's condition was successfully rescued. Antibodies for human T-cell lymphotropic virus-1 (HTLV-1) were positive. HTLV-1 infection and the administration of corticosteroids are known risk factors for strongyloides hyperinfection syndrome. Therefore, physicians should consider this disease in the differential diagnosis of patients from endemic areas who present with gastrointestinal symptoms under these risk factors.
\end{abstract}

Key words: Strongyloides stercoralis, strongyloides hyperinfection syndrome, acute respiratory distress syndrome, human T-cell lymphotropic virus-1, corticosteroid, ivermectin

(Intern Med 54: 83-87, 2015)

(DOI: 10.2169/internalmedicine.54.3284)

\section{Introduction}

Strongyloides stercoralis (S. stercoralis) is an intestinal helminth that infects humans transdermally. The prevalence of strongyloidiasis is high in tropical and subtropical regions. Although Okinawa prefecture and the southwest area of Kagoshima prefecture in Japan were known to be endemic areas for this worm in the past, the rate of infection has dramatically decreased in association with improvements in hygiene. A unique characteristic of S. stercoralis is its ability to reinfect the human body, called autoinfection. This looped lifecycle enables the parasite to live in the human body for extended periods of time. The administration of corticosteroids and human T-cell lymphotropic virus-1 (HTLV-1) infection are major risk factors exacerbating $S$. stercoralis infection. We herein report a case of acute respiratory distress syndrome (ARDS) due to strongyloides hyperinfection syndrome in a HTLV-1 carrier receiving corticosteroid therapy.

\section{Case Report}

A 62-year-old woman with cervical cancer (stage IIIb) was admitted to our hospital for concurrent chemoradiation therapy. The chemotherapy regimen included the administration of cisplatin/paclitaxel (63.5 mg/body for both drugs) on day 1 followed by paclitaxel alone on days 8 and 15. High doses of steroids (16.5 $\mathrm{mg}$ of dexamethasone on days 1,8 and 15 with $13.2 \mathrm{mg}$ of dexamethasone on day 2) were administered along with the chemotherapy. The initial planned protocol for radiation treatment included external irradiation at a dose of 50 Gy in 25 fractions and intracavitary irradiation at a dose of $18 \mathrm{~Gy}$ in three fractions. The second day after the initiation of therapy, the patient developed diarrhea. Although the dose of chemotherapy was completed on day 1 of the second cycle, further therapy was discontinued due to the development of a fever, refractory diarrhea and nausea. Seven weeks after the start of chemotherapy, the patient presented with melena, and supportive care was provided under

\footnotetext{
${ }^{1}$ Department of Infectious Diseases, Respiratory and Digestive Medicine, Faculty of Medicine, University of the Ryukyus, Japan, ${ }^{2}$ Department of Obstetrics and Gynecology, Faculty of Medicine, University of the Ryukyus, Japan, ${ }^{3}$ Department of Pathology and Oncology, Faculty of Medicine, University of the Ryukyus, Japan and ${ }^{4}$ Department of Anesthesiology, Faculty of Medicine, University of the Ryukyus, Japan Received for publication May 15, 2014; Accepted for publication August 6, 2014 Correspondence to Dr. Takeshi Kinjo, kumanomis6@yahoo.co.jp
} 


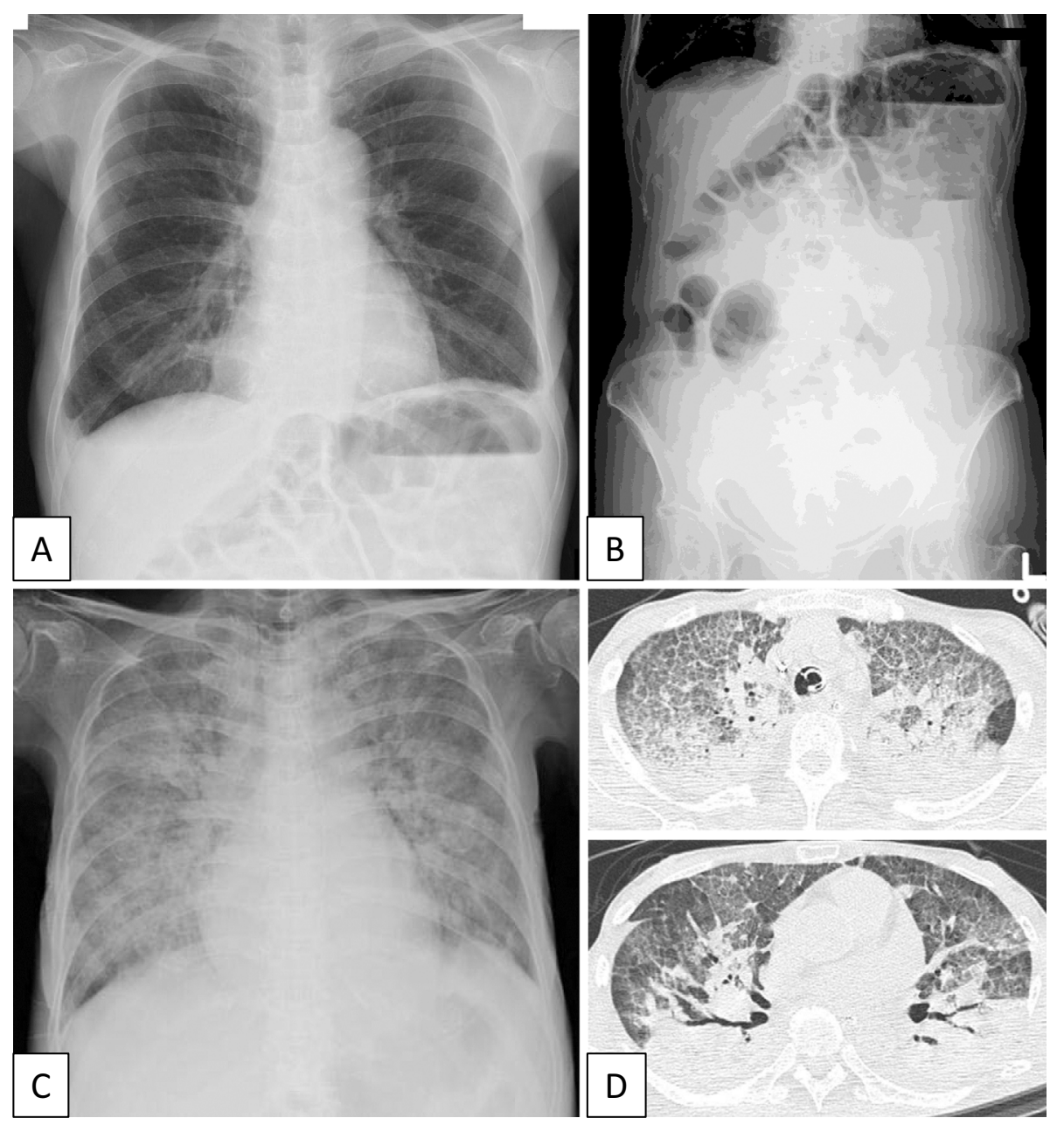

Figure 1. Radiological images in the present case. Chest and abdominal X-rays obtained five days before ICU admission (A, B). A: A chest X-ray showed small bilateral pleural effusion. B: An abdominal $X$-ray revealed excessive intestinal gas and air-fluid levels in the left upper quadrant. A chest X-ray and CT scan obtained on the day of ICU admission (C, D). C: A chest X-ray showed bilateral diffuse infiltration. A chest $\mathrm{CT}$ scan revealed massive consolidation, thickening of the interlobular septa and traction bronchiectasis (D).

a diagnosis of radiation colitis on colon fiberscopy. One week later, she began to vomit, and an abdominal X-ray revealed excessive gas and air-fluid levels in the left upper quadrant. A chest X-ray obtained on the same day showed small bilateral pleural effusion (Fig. 1A, B). The main laboratory findings at that point were as follows: white blood cell count $=5,900 / \mu \mathrm{L}$ (neutrophils: $82.9 \%$, lymphocytes: $9.5 \%$, eosinophils: $3.2 \%$ ), hemoglobin $=9.2 \mathrm{~g} / \mathrm{dL}$, platelet count $=24.9 \times 10^{4} / \mu \mathrm{L}$, albumin $=1.5 \mathrm{mg} / \mathrm{dL}$, blood urea nitrogen $=3 \mathrm{mg} / \mathrm{dL}$, creatinine $=0.34 \mathrm{mg} / \mathrm{dL}$, total bilirubin $=$ $0.3 \mathrm{mg} / \mathrm{dL}$, aspartate aminotransferase $=16 \mathrm{U} / \mathrm{L}$, alanine aminotransferase $=11 \mathrm{U} / \mathrm{L}$, lactic dehydrogenase $=215 \mathrm{U} / \mathrm{L}$, C-reactive protein $=8.98 \mathrm{mg} / \mathrm{dL}, \mathrm{Na}=135 \mathrm{mEq} / \mathrm{L}, \mathrm{K}=2.3$ $\mathrm{mEq} / \mathrm{L}, \mathrm{Cl}=92 \mathrm{mEq} / \mathrm{L}$. The patient was therefore diagnosed with ileus and observed with an indwelling nasogastric tube. Five days later, she began to complain of dyspnea and required oxygen administration using a nasal cannula. Her respiratory condition gradually worsened, and seven days after the diagnosis of ileus, she exhibited a decreased level of consciousness, low blood pressure and worsening of her respiratory status, requiring that she be transported to the intensive care unit (ICU). A chest X-ray showed bilateral diffuse infiltration and a chest $\mathrm{CT}$ scan revealed massive consolidation, interlobular septal thickening and traction bronchiectasis (Fig. 1C, D). Echocardiography indicated negative findings for both cardiac failure and fluid overload. Diffuse intrabronchial bloody discharge was observed on bronchoscopy, and the right middle lobe was subsequently washed with $10 \mathrm{~mL}$ of normal saline (Fig. 2). A pathological examination revealed numerous nematodes identified as S. stercoralis based on their morphological characteristics (Fig. 3). We thus diagnosed her with strongyloides hyperinfection syndrome and initiated treatment with ivermectin as well as broad spectrum antibiotics. Streptococcus gordonii and Klebsiella pneumoniae were also detected in a blood culture. Thereafter, the ivermectin was administered daily until nematodes were no longer detected in the sputum. As a result, the patient's condition gradually improved, and she 


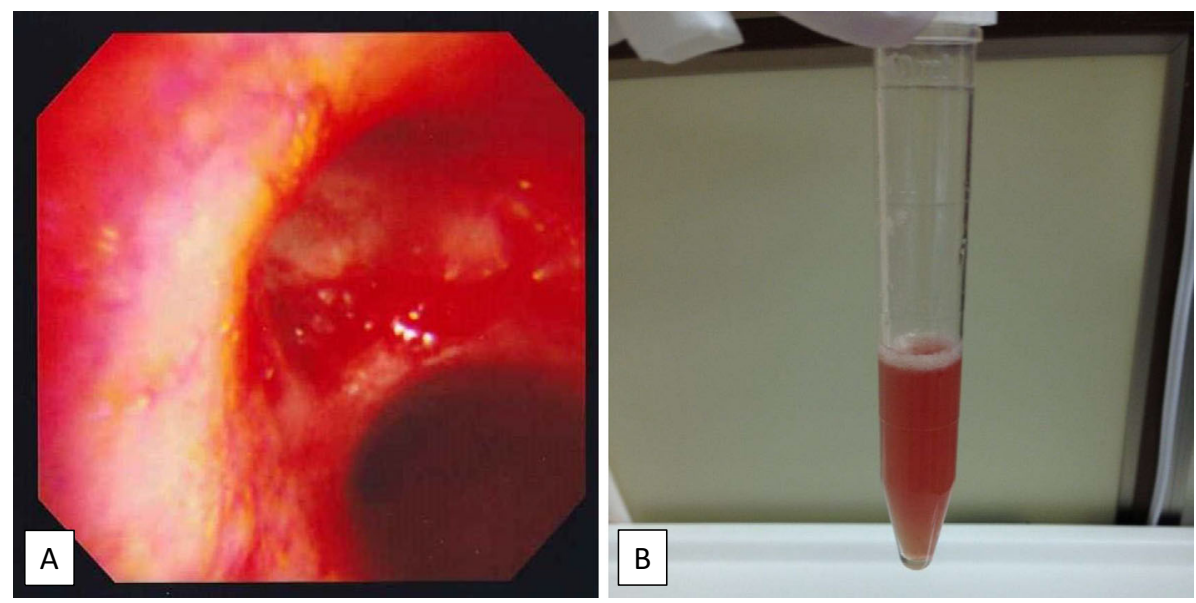

Figure 2. Bronchoscopic view and appearance of the lavage fluid. A: Diffuse intrabronchial bloody discharge was observed on bronchoscopy. B: Bloody lavage fluid was collected from the patient.

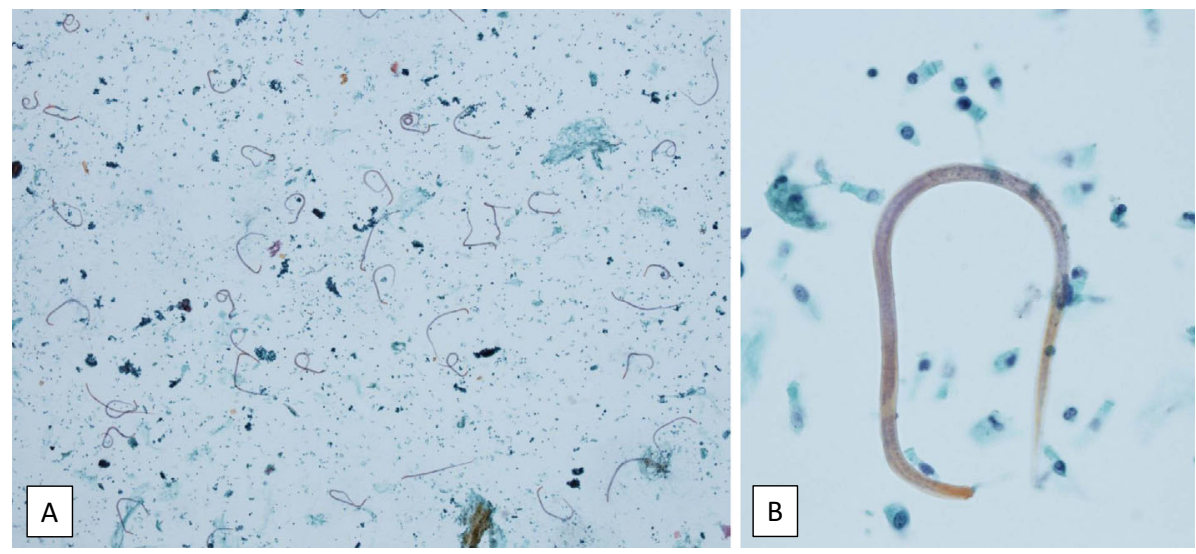

Figure 3. Microscopic view of the lavage fluid. A: The lavage fluid showed numerous nematodes on Papanicolaou staining $(\times 20)$. B: The nematodes were identified to be filariform larvae of $S$. stercoralis based on their morphological characteristics $(\times 400)$.

moved to the general ward on 19th day in the ICU (Fig. 4). Potential HTLV-1 infection, which is known to be a major risk factor contributing to the development of strongyloides hyperinfection syndrome, was assessed following ICU admission; the results were positive.

\section{Discussion}

S. stercoralis is an intestinal helminth that infects humans via the skin when in contact with contaminated soil. Filariform larvae penetrate the skin and subsequently travel to the lungs hematogenously followed by the gastrointestinal tract and sputum, becoming female adult worms in the small intestine. The adult female worms then produce eggs, which hatch to form rhabditiform larvae that are ultimately excreted in the feces. The notable feature of this worm is that some rhabditiform larvae can transform into infectious filariform larvae while still in the body. Such larvae have the capacity to reinvade the vessels and consequently return to the lungs. This phenomenon is called autoinfection and enables the parasite to live within the human body for a long period of time (1). The prevalence of strongyloidiasis is high in tropical and subtropical regions. Although Okinawa prefecture and the southwest area of Kagoshima prefecture in Japan were previously known to be regions endemic for this worm, the rate of infection has decreased drastically due to improvements in hygiene (2). According to a recent epidemiological study in Okinawa (3), the prevalence of S. stercoralis infection is extremely low (approximately 0-1\%) in generations born after 1950, which includes the present patient.

The current patient presented with diarrhea and vomiting, the typical symptoms of $S$. stercoralis infection; however, eosinophilia was absent prior to ICU admission. A recent report described the detection of eosinophilia in $90 \%$ of patients with $S$. stercoralis infection, although eosinophilia is often absent in patients with an immunosuppressive status $(4,5)$. The lack of eosinophilia is considered to be one encumbrance delaying a correct diagnosis in this case.

Major risk factors contributing to the development of strongyloides hyperinfection syndrome include the administration of corticosteroids and HTLV-1 infection $(1,2,6)$. In 


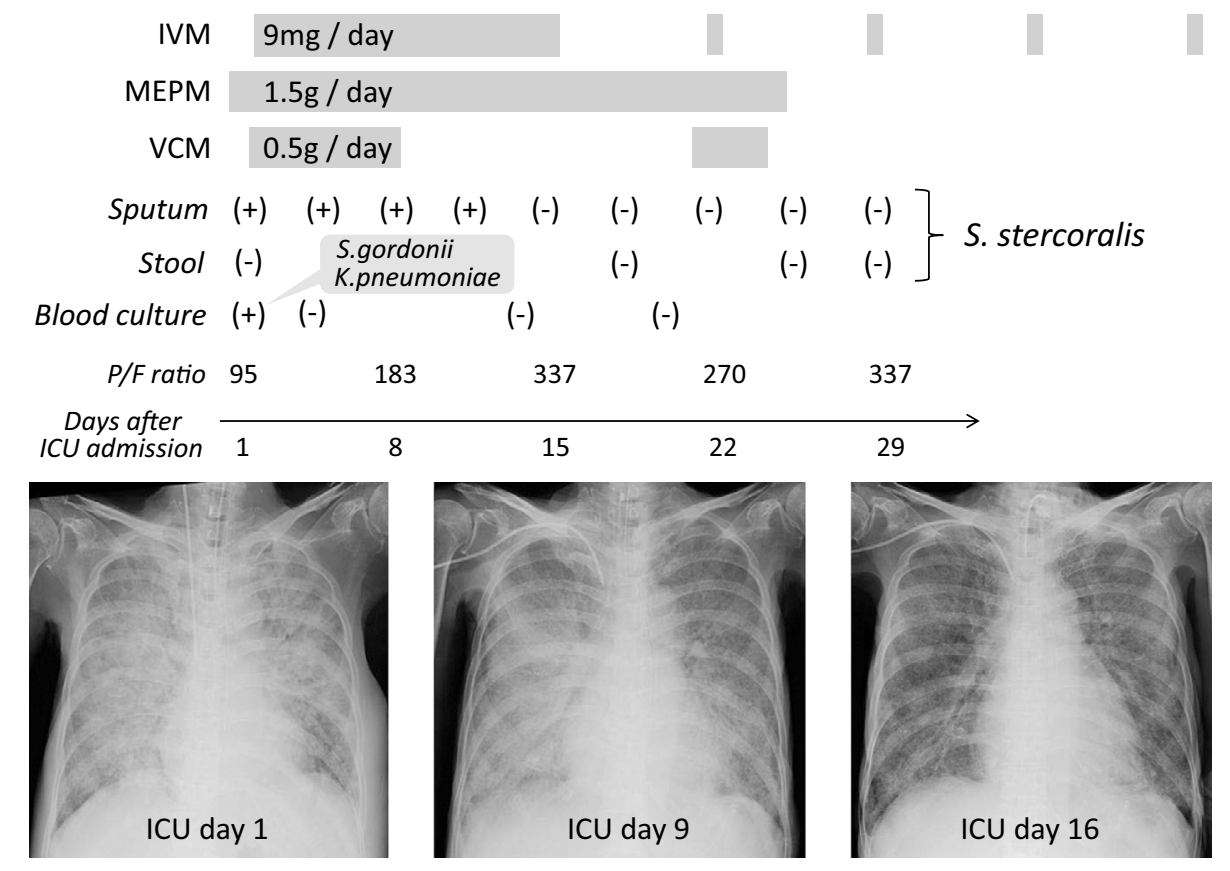

Figure 4. Clinical course after ICU admission. Ivermectin was administered daily until nematodes in the sputum became negative, followed by four doses of weekly treatment. Streptococcus gordonii and Klebsiella pneumoniae were also detected in a blood culture, and meropenem injection was continued for more than three weeks. The $\mathrm{PaO}_{2} / \mathrm{FiO}_{2}$ ratio gradually improved, and the patient moved to the general ward on the 19th day in the ICU.

the present case, the patient was both an HTLV-1 carrier and received high doses of corticosteroids during chemotherapy. Although the mechanisms underlying the susceptibility of HTLV-1 carriers to $S$. stercoralis infection are not fully understood, a suppressed immune response, such as that involving low levels of eosinophils, IgE and IL-5 and a higher count of regulatory $\mathrm{T}$ cells in the blood among HTLV-1positive patients, has been reported $(7,8)$.

The first case of ARDS caused by $S$. stercoralis infection was reported in 1987 (9), and there have since been several reports showing that strongyloides may induce respiratory failure. Most reports have described the condition to be lethal with a very high rate of mortality (10-13). The present case is considered to be valuable in that the patient was successfully rescued with the daily administration with ivermectin. Although the treatment protocol for strongyloides hyperinfection syndrome is not yet established, the use of daily dosing with ivermectin until two weeks after the confirmed disappearance of the worm in the stool is recommended $(1,14)$. In the current case, the duration of ivermectin therapy was determined based on the disappearance of the worm in the patient's sputum, as S. stercoralis was not detected in the stool.

In conclusion, we herein reported a case of ARDS caused by strongyloides hyperinfection syndrome. Although the prevalence of $S$. stercoralis infection has recently decreased, physicians must consider this parasitic infection in the differential diagnosis when treating patients complaining of gastrointestinal symptoms of unknown etiology, especially in cases involving HTLV-1 carriers and corticosteroid users.
The authors state that they have no Conflict of Interest (COI).

\section{References}

1. Greaves D, Coggle S, Pollard C, Aliyu SH, Moore EM. Strongyloides stercoralis infection. BMJ 347: f4610, 2013.

2. Zaha O, Hirata T, Kinjo F, Saito A. Strongyloidiasis--progress in diagnosis and treatment. Intern Med 39: 695-700, 2000.

3. Aoyama H, Hirata T, Sakugawa H, et al. An inverse relationship between autoimmune liver diseases and Strongyloides stercoralis infection. Am J Trop Med Hyg 76: 972-976, 2007.

4. Valerio L, Roure S, Fernández-Rivas G, et al. Strongyloides stercoralis, the hidden worm. Epidemiological and clinical characteristics of 70 cases diagnosed in the North Metropolitan Area of Barcelona, Spain, 2003-2012. Trans R Soc Trop Med Hyg 107: 465-470, 2013.

5. Greaves D, Gouliouris T, O’Donovan M, Craig JI, Török ME. Strongyloides stercoralis hyperinfection in a patient treated for multiple myeloma. Br J Haematol 158: 2, 2012.

6. Verdonck K, González E, Van Dooren S, Vandamme AM, Vanham G, Gotuzzo E. Human T-lymphotropic virus 1: recent knowledge about an ancient infection. Lancet Infect Dis 7: 266-281, 2007.

7. Hirata T, Uchima N, Kishimoto K, et al. Impairment of host immune response against strongyloides stercoralis by human $\mathrm{T}$ cell lymphotropic virus type 1 infection. Am J Trop Med Hyg 74: 246-249, 2006.

8. Montes M, Sanchez C, Verdonck K, et al. Regulatory T cell expansion in HTLV-1 and strongyloidiasis co-infection is associated with reduced IL-5 responses to Strongyloides stercoralis antigen. PLoS Negl Trop Dis 3: e456, 2009.

9. Cook GA, Rodríguez H, Silva H, Rodríguez-Iturbe B, Bohorquez de Rodríguez $\mathrm{H}$. Adult respiratory distress secondary to strongyloidiasis. Chest 92: 1115-1116, 1987.

10. Livneh A, Coman EA, Cho SH, Lipstein-Kresch E. Strongyloides 
stercoralis hyperinfection mimicking systemic lupus erythematosus flare. Arthritis Rheum 31: 930-931, 1988.

11. Thompson JR, Berger R. Fatal adult respiratory distress syndrome following successful treatment of pulmonary strongyloidiasis. Chest 99: 772-774, 1991.

12. Setoyama M, Fukumaru S, Takasaki T, Yoshida H, Kanzaki T. SLE with death from acute massive pulmonary hemorrhage cause by disseminated strongyloidiasis. Scan J Rheumatol 26: 635-640,
1997.

13. Hauber HP, Galle J, Chiodini PL, et al. Fatal outcome of a hyperinfection syndrome despite successful eradication of Strongyloides with subcutaneous ivermectin. Infection 33: 383-386, 2005.

14. Mejia R, Nutman TB. Screening, prevention, and treatment for hyperinfection syndrome and disseminated infections caused by Strongyloides stercoralis. Curr Opin Infect Dis 25: 458-463, 2012.

(C) 2015 The Japanese Society of Internal Medicine http://www.naika.or.jp/imonline/index.html 